\title{
Segmentation in vertebrates: clock and gradient finally joined
}

\author{
Alexander Aulehla ${ }^{1}$ and Bernhard G. Herrmann ${ }^{2}$ \\ Max-Planck-Institute for Molecular Genetics, Department of Developmental Genetics, 14195 Berlin, Germany
}

\begin{abstract}
The vertebral column is derived from somites formed by segmentation of presomitic mesoderm, a fundamental process of vertebrate embryogenesis. Models on the mechanism controlling this process date back some three to four decades. Access to understanding the molecular control of somitogenesis has been gained only recently by the discovery of molecular oscillators (segmentation clock) and gradients of signaling molecules, as predicted by early models. The Notch signaling pathway is linked to the oscillator and plays a decisive role in inter- and intrasomitic boundary formation. An Fgf8 signaling gradient is involved in somite size control. And the (canonical) Wnt signaling pathway, driven by Wnt3a, appears to integrate clock and gradient in a global mechanism controlling the segmentation process. In this review, we discuss recent advances in understanding the molecular mechanism controlling somitogenesis.
\end{abstract}

In amniotes, the presomitic mesoderm (psm) arises in the primitive streak and at later stages in the tail bud located at the caudal end of the embryo. These structures function as "growth zones" that provide proliferating cells, which are recruited to the three germ layers, thereby elongating the body anlage. The psm separates into two halves (segmental plates in chick), eventually forming two rows of spheres of epithelial cells, the somites, which are located on either side of the neural tube (Fig. 1). They give rise to the vertebrae, all striated muscles, and several other tissues (Christ and Ordahl 1995).

\section{Periodicity and directionality}

Somites bud off in pairs from the anterior ends of the two psm halves. In normal development, they are perfectly aligned and form a regular array along the anterior-pos-

[Keywords: Somitogenesis; segmentation clock; gradients in segmentation; Wnt signaling; Notch signaling; FGF signaling]

${ }^{1}$ Present address: Department of Internal Medicine, University Hospital Basel, CH-4031 Basel, Switzerland

${ }^{2}$ Corresponding author.

E-MAIL herrmann@molgen.mpg.de; FAX 49-30-8413-1229.

Article and publication are at http://www.genesdev.org/cgi/doi/10.1101/ gad. 1217404 terior $(\mathrm{A}-\mathrm{P})$ axis. Somite formation takes place periodically in a fixed anterior-to-posterior sequence.

In the chick embryo, a new somite is formed approximately every $90 \mathrm{~min}$, whereas in the mouse embryo, the periodicity varies, dependent on the axial position (Tam 1981). Classical embryology experiments revealed that periodicity and directionality of somite formation are controlled by an intrinsic program set off in the cells as they are recruited into the psm. For instance, when the psm is inverted rostro-caudally, somite formation in the inverted region proceeds from caudal to rostral, maintaining the original direction (Christ et al. 1974). Moreover, neither the transversal bisection nor the isolation of the psm from all surrounding tissues stops the segmentation process. Segments form at the right time in the right place (Packard 1978; Palmeirim et al. 1998).

\section{Somite number control}

Another striking experimental finding concerned the regular spacing of somites. When Xenopus laevis embryos were experimentally reduced in size, the somite size was adjusted while the normal number was maintained (Cooke 1975). Likewise, in the mouse mutant amputated, the body length is reduced, but the somite number is normal and somites are smaller at day $9.5 d p c$ (Flint et al. 1978; Tam 1981). In fact, there is very little variability in total somite number within a species. The tight control of somite number implies a global regulation of somite size: The final body size is integrated into the formation of each individual somite early during development.

\section{Early models}

These experimental results and observations stimulated the development of theoretical models that attempted to explain the nature of the mechanism underlying somitogenesis (Cooke and Zeeman 1976; Meinhardt 1986; Kerszberg and Wolpert 2000; for review, see Dale and Pourquie 2000). Several of these models involve an oscillator or segmentation clock, which is acting within each individual cell of the psm. The oscillator was proposed to function as a pacemaker, thus accounting for the intrinsic periodicity of somitogenesis.

The second important component of the control mechanism is constituted by a morphogen gradient 


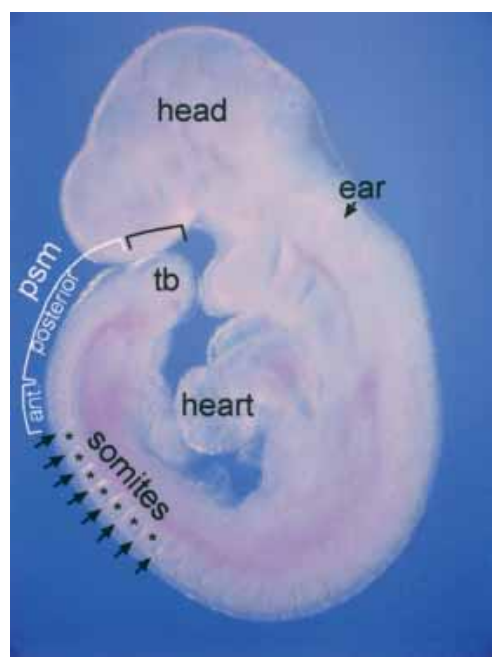

Figure 1. E9.5 mouse embryo. Arrows indicate somite boundaries, asterisks indicate somites (only caudal somites are labeled). (Psm) Presomitic mesoderm, divided in anterior and posterior territories; $(\mathrm{tb})$ tail bud.

(Meinhardt 1986) or, more vaguely, a gradient of developmental cell change (the "wave front" in the clock and the wave front model; Cooke and Zeeman 1976). Morphogen gradients have been proposed as mechanisms for conferring positional information to cells (Wolpert 1989). According to Meinhardt (1986), a morphogen gradient could account for somite number control under several premises: first, the slope of the gradient determines somite size. The steeper the slope, the fewer cells fall below a threshold within a defined period of time, resulting in smaller somites. Second, the slope of the gradient depends on the field size (e.g., the length of the $\mathrm{psm})$. The smaller the field, the steeper must be the gradient, if the absolute morphogen concentration remains unchanged.

Remarkably, some three decades after these models were proposed, the functional importance of clock and gradient in the segmentation process have been confirmed experimentally.

\section{Notch signaling in the clock mechanism}

The involvement of the Notch signaling pathway in somitogenesis was first revealed by the finding that Notch1 and its ligand Dll1, which are highly expressed in the psm of mouse embryos, play important roles in inter- and intrasomitic boundary formation (Conlon et al. 1995; Hrabe de Angelis et al. 1997). In 1997, the discovery of a molecular oscillator was reported (Palmeirim et al. 1997). It was identified by the observation that the expression of c-hairy1 mRNA in the psm of chick embryos oscillates with the same periodicity as the somites form. C-hairy1 is a target of the Notch signaling cascade. Subsequently, additional cyclic genes, such as Lunatic fringe (Lfng), Hes7, and Hes1 were identified in different species (Evrard et al. 1998; Forsberg et al. 1998; McGrew et al. 1998; Zhang and Gridley 1998; Aulehla and Johnson 1999; Jouve et al. 2000; Bessho et al. 2001; Hirata et al. 2002; for review, see Bessho and Kageyama 2003). All of them are linked to the Notch signaling pathway (hereafter termed "cyclic Notch targets"), and their mRNA oscillations are all in phase (Aulehla and Johnson 1999; Barrantes et al. 1999; Jouve et al. 2000; Bessho et al. 2001). Lfng and Hes7 are directly activated by Notch signaling (Bessho et al. 2001; Cole et al. 2002; Morales et al. 2002). Promoter elements required for oscillating Lfng expression were identified and shown in vivo to depend on Notch signaling (Cole et al. 2002; Morales et al. 2002). These data indicated that Notch-signaling activity in the psm is itself oscillating and is either controlled by the segmentation clock or is a central component of it.

At this point, it is important to add that only the posterior part of the psm and the tail bud show oscillating Notch signaling activity, whereas the anterior region, in which segment boundaries actually form, displays stable Notch target gene expression.

Recently it has been proposed that a negative feedback loop in the Notch signal cascade, established via Lfng, may underlie the segmentation clock (Dale et al. 2003). When Lfng is ectopically expressed at high levels in the segmental plate of chick embryos, cyclic expression of endogenous $L f n g$ is lost and the segmentation phenotype resembles loss of Notch signaling mutations. Continuous (noncyclic) overexpression of Lfng in transgenic mouse embryos, however, did not impair oscillations of endogenous $L f n g$, whereas segmentation was clearly affected under these conditions (Serth et al. 2003). The discrepancy between these results could be related to different experimental design; more experiments are needed to establish the precise role of Lfng in the control of Notch receptor activity and in the segmentation process.

An alternative explanation for the generation of periodic Notch target gene activation has been put forward by others (Bessho et al. 2003). Hes7 is a transcriptional regulator and direct target of Notch signaling. It is not only transcribed in an oscillating manner, but the Hes7 protein also appears and disappears periodically during the segmentation cycle (Bessho et al. 2001, 2003). Interestingly, the regions of de novo mRNA and protein synthesis are mutually exclusive. Indeed, Hes7 protein was shown to repress Hes7 transcription and that of other cyclic Notch targets. In addition, Hes7 protein is highly unstable. Thus, Hes7 might control periodic transcription of Notch targets via a negative feedback mechanism. On the basis of these data, a Hes7 negative feedback loop was suggested as molecular basis of the segmentation clock (Bessho et al. 2003).

It is now well established that Notch signaling plays an important role in the clock mechanism. However, somites still form when Notch signaling is impaired or abolished, suggesting that additional factors must be involved. For instance, in mouse embryos lacking RBP-J к (CBF1), the effector of Notch activity in the nucleus, somites do form albeit in a delayed and irregular manner 
(Oka et al. 1995). Thus, either compensatory mechanisms exist or the Notch pathway is not the key control element of the segmentation clock.

\section{Wnt signaling in the clock}

The finding of our group that Axin2, a negative regulator of the Wnt signaling cascade (Zeng et al. 1997; Behrens et al. 1998; Yamamoto et al. 1998), shows oscillatory mRNA expression in the psm provided the first hint that an additional pathway is linked to the segmentation clock (Aulehla et al. 2003). This link is particularly interesting, because Wnt signaling is essential for the elongation of the embryonic body and for the formation of psm (Takada et al. 1994; Greco et al. 1996; Galceran et al. 1999; Liu et al. 1999). It was previously shown in vitro that Axin2 is a direct target of the Wnt signaling cascade (Jho et al. 2002; Lustig et al. 2002). Mutagenesis of Lef/ Tcf sites in the Axin2 promoter followed by reporter assays in mouse embryos confirmed that Axin2 expression in vivo in the psm and tail bud also directly depends on the canonical Wnt pathway (Aulehla et al. 2003). In addition, Axin2 is strongly down-regulated in embryos homozygous for the Wnt3a hypomorphic allele vestigial tail (vt; Aulehla et al. 2003).

These data led us to propose a negative feedback mechanism between Wnt3a and its inhibitor Axin2 in the psm. The fact that Axin2 transcription oscillates implies that both Axin2 RNA and protein must be highly unstable, thus allowing periodic switching of Wnt signaling between active and inactive states (Aulehla et al. 2003). Instability of Axin2 mRNA is obvious from the expression pattern. Evidence for instability of Axin2 protein comes from the observation that the Axin2 homolog Axin is destabilized by dephosphorylation, which can be triggered by Wnt signaling (for reviews, see Seidensticker and Behrens 2000; Tolwinski and Wieschaus 2004). The combined data suggest that in the psm, Wnt3a produces regular oscillations of Wnt signaling activity inside the cells through a negative feedback loop involving its target Axin2 and subsequent destabilization of the Axin2 protein.

\section{Wnt and Notch signaling pathways interact in the psm}

The existence of two signal cascades, each producing oscillations of target gene activity as a result of negative feedback mechanisms and each linked to the segmentation clock, poses the question of whether they exist in parallel or interact. Parallel in situ expression analysis showed that Lfng and Axin2 are alternately up- and down-regulated in the posterior psm of embryonic day 9.5 (E9.5) mouse embryos, demonstrating that the Notch and Wnt signaling cascades are alternately active in the psm, with some degree of overlap. In $D 111^{-/-}$embryos, in which Notch signaling is impaired, Axin2 transcription occurs and oscillations of Axin2 mRNA can still be detected, though the pattern differs from wild type (Aulehla et al. 2003).
In contrast, when Wnt signaling in the psm is strongly down-regulated or abolished, as visualized by lack of Axin2 mRNA in $v t / v t$ embryos, oscillations of Lfng expression are abolished as well (Aulehla et al. 2003). Importantly, the territory of stable Lfng expression in the anterior psm is not affected.

The combined data demonstrate a tight link between the Wnt and Notch signal cascades in the oscillating part of the psm, and suggest that the oscillations of Notch signaling activity are dependent on Wnt3a.

Evidence from the fruit fly has demonstrated multiple genetic interactions between the Wnt/wg and Notch signaling pathways in several developmental contexts (Ruel et al. 1993; Axelrod et al. 1996; Martinez Arias 1998; Fanto and Mlodzik 1999). In addition, there is evidence for direct protein interaction between dsh, an intracellular component of the Wnt/wg signaling cascade, and Notch (Axelrod et al. 1996). Moreover, in mouse cells, GSK3 $\beta$, a negative regulator of $\beta$-catenin, is able to bind and phosphorylate the intracellular domain of Notch1, thereby modulating its activity (Foltz et al. 2002). These observations suggest possible molecular links for the cross-talk between the Wnt and Notch signaling pathways in the psm.

\section{The FGF8 gradient}

The second central component of the mechanism controlling stepwise setting of the segment boundary position was predicted by early models to consist either of a "wave front," a maturation wave moving in A-P direction, or of a graded morphogen peaking at the caudal end of the embryo (Cooke and Zeeman 1976; Meinhardt 1986). The latter turned out to match reality more closely. Fgf8 RNA was shown to form a gradient along the psm (Dubrulle et al. 2001). Strikingly, by increasing the local concentration of Fgf8 protein in the psm, the somite size was reduced and inhibition of FGF signaling resulted in larger somites, just as predicted by theoretical considerations (Dubrulle et al. 2001). Recently, the same group showed that de novo mRNA production of Fgf8 is restricted to the tail bud (Dubrulle and Pourquie 2004). They used qPCR and immunohistochemistry to demonstrate a graded distribution of both Fgf 8 mRNA and protein along the psm and concluded that the mRNA gradient of Fgf8 may be formed by a decay mechanism (Dubrulle and Pourquie 2004). These data clearly demonstrated the importance of Fgf8 in determining the position at which the segment boundary will form. What remained unresolved was the question of how the FGF signaling pathway is linked to the segmentation clock.

\section{The Wnt gradient}

Evidence for a second gradient along the psm involved in setting the segment boundary position has been provided by our group (Aulehla et al. 2003). Axin2 RNA was shown to be expressed in a graded manner along the psm, 
peaking in the tail bud. As mentioned earlier, in the posterior psm and tail bud, Axin2 is directly activated by Wnt signaling. These data together provide indirect evidence for graded Wnt signaling activity along the psm. $W n t 3 a$ is only expressed in the primitive streak and tail bud, located at the posterior tip of the embryo. Because protein production must be restricted to this region, a mechanism sufficient for establishing a gradient of Wnt3a protein can be envisioned. Cells leaving the tail bud stop de novo production of Wnt3a protein; hence, over time the Wnt3a concentration in the cellular environment should drop. At the same pace, the cell will take a more and more anterior position in the psm, while the embryo is elongating caudally and the tail bud is moving away from the cells that it is producing. Finally, a threshold level of Wnt3a is reached and the cell is ready to become part of a somite (Aulehla et al. 2003). A similar mechanism was recently suggested to act in the formation of the Fgf8 RNA and protein gradients along the psm (Dubrulle and Pourquie 2004).

The importance of Wnt3a protein in setting the segment boundary position was demonstrated by increasing the local concentration of Wnt3a protein in the psm. As shown for Fgf8, smaller somites, occasionally in combination with anterior shift of the segment boundary, formed at the site of increased Wnt3a concentration (Aulehla et al. 2003). In addition, experimental downregulation of Wnt signaling through continuous expression of Axin2 in the psm from a transgene construct resulted in larger somites. Lfng was ectopically expressed in the psm of transgenic embryos, suggesting that inhibition of Wnt signaling by Axin2 resulted in ectopic up-regulation of Notch signaling activity. These data provided additional evidence for a link between the Wnt and Notch signaling cascades (Aulehla et al. 2003).

In summary, the experimental evidence strongly suggests the existence of a Wnt3a protein gradient along the psm, which plays a decisive role in setting the segment boundary position. How are the Wnt3a and Fgf8 gradients related to each other?

The expression of Fgf8 RNA is strongly down-regulated in the tail buds of $v t / v t$ embryos, suggesting that Fgf8 expression depends on Wnt3a (Aulehla et al. 2003). Thus, Wnt3a appears to control the formation of the Fgf8 gradient in an indirect manner. But why two parallel gradients? One intriguing possibility is that FGF signaling may enhance Wnt signaling. FGF acts via two different signaling cascades, the MAP kinase and the PI-3 kinase pathways. The latter can up-regulate Akt (PKB) activity which is known to inhibit Gsk3 $\beta$ (Jun et al. 1999; Fukumoto et al. 2001). Gsk3 $\beta$ is an inhibitor of the Wnt signal cascade that binds to Axin/Axin2 and phosphorylates $\beta$-catenin, making it accessible for protein degradation. Thus, there is a link between the FGF and the Wnt signaling cascades through Akt and Gsk3 $\beta$. Indeed, it was recently shown that phosphorylated (activated) Akt is graded along the psm in parallel to the Fgf8 gradient, whereas no clear graded distribution of phosphorylated MAPK (mitogen-activated protein kinase) was observed (Dubrulle and Pourquie 2004). Further experiments are needed to explore the relationship of the Fgf8 and Wnt signaling gradients in the psm in more detail.

\section{Gradient and clock are joined through Wnt3a}

Axin2 is the first gene found to be linked to both central components controlling segmentation, the oscillator and the gradient. It is a direct target of the Wnt3a gradient and at the same time shows oscillating transcription with the same periodicity as the segmentation process. All other genes known to act in somitogenesis are either linked to the clock or the gradient, but not to both. In addition, Wnt3a controls oscillating Notch activity in the psm and Fgf8 expression in the tail bud. Therefore, Wnt3a and the Wnt signaling cascade must play a central role in the segmentation clock and in the gradient controlling somitogenesis.

We have proposed a model for the molecular control of the segmentation mechanism, in which the Wnt3a gradient provides the force driving the segmentation clock (Aulehla et al. 2003; see Figs. 2, 3). Cells exposed to higher-than-threshold levels of Wnt3a go on oscillating (clock on), whereas neighboring (more anterior) cells exposed to lower levels stop the clock because of insufficient Wnt signaling activity (clock off). This novel link between the morphogen gradient and the clock provides a plausible explanation for how the gradient is translated into the stepwise formation of segments: Activation of the "Wnt on" phase of the clock cycle creates at the threshold position an interface between neighboring "clock on" and "clock off" states, setting the boundary position. The periodicity of the clock cycle in combination with continuous posterior "moving" of the threshold ensures setting of the boundary position at regular intervals.

Notch is known to play an important role in boundary formation, and Lfng is a modifier of the Notch receptor required for Notch activation and signaling (Haines and Irvine 2003). Evidence in the chick has shown that under particular experimental conditions, Lfng is able to induce an ectopic segment boundary within a somite (Sato et al. 2002). Thus, Lfng is a good candidate for a clockcontrolled factor involved in somite boundary formation. During the clock cycle, $L f n g$ expression is up-regulated along the psm and stabilized in the anterior psm, while transcripts disappear from the posterior psm and tail bud. The region showing stable expression has a sharp boundary to more anterior cells, which are Lfng negative. This expression boundary is likely to form an interface at which somite boundary formation may be induced downstream of Notch (Sato et al. 2002).

The process of boundary formation itself is complex and involves a large number of genes, as reviewed elsewhere (Saga and Takeda 2001; Iulianella et al. 2003).

Thus, Wnt3a may indirectly control the expression of Lfng in the posterior, oscillating region of the psm, up to the position where the future segment boundary is induced, by controlling oscillating Notch signaling activity in the segmentation clock. Wnt signaling may be enhanced by Fgf8 signaling, the latter probably being im- 
A

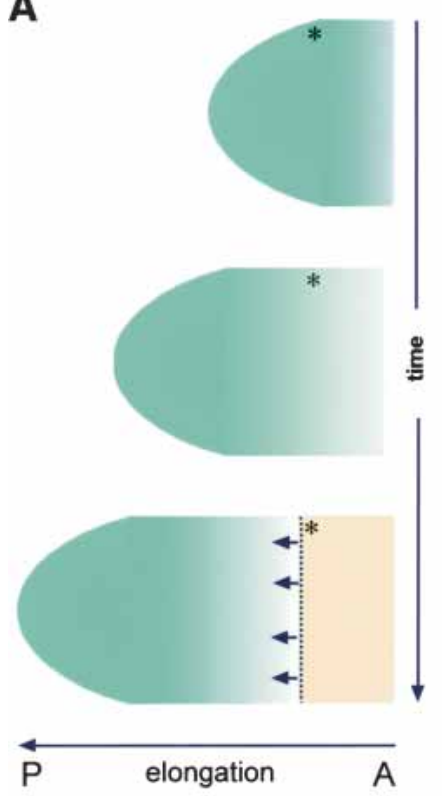

B

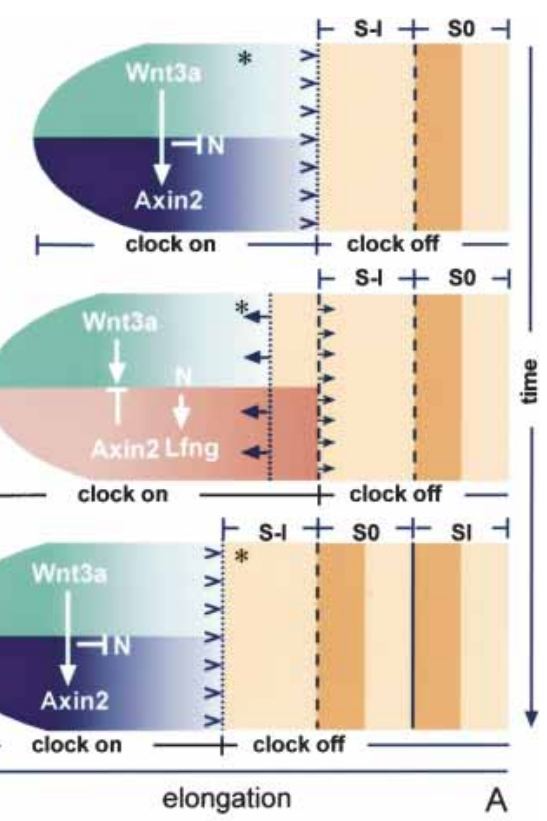

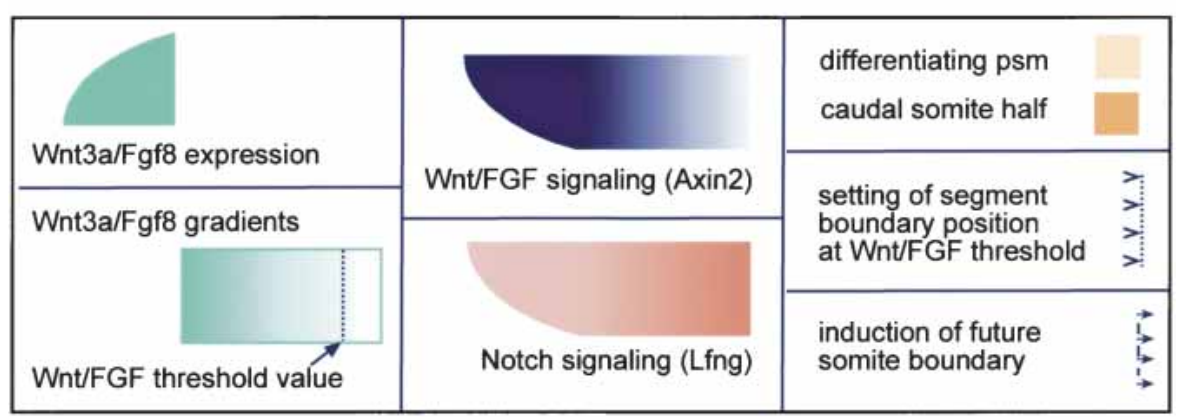

Figure 2. Clock and gradient model. (A) Formation of the gradient. The caudal end of the embryo (tail bud) provides cells and Wnt3a protein (green). Because of posterior growth, the source of the signal moves away from the cells (indicated by an asterisk) that it produced. Decay of the signal molecules in the psm over time forms a morphogen gradient. Cells exposed to levels below a threshold value start differentiation (yellow). The threshold position "moves" in rostro-caudal direction (arrows). The Fgf8 gradient is established in a similar manner: Fgf8 RNA is expressed under control of Wnt3a at the caudal end, an RNA gradient is established by decay, and RNA translation produces a gradient of Fgf8 signal. Fgf8 signaling may enhance Wnt signaling, which would be important for the range in which the latter is active. $(B)$ The gradient drives the clock. Cells exposed to higher-than-threshold values of Wnt3a activate the oscillator (clock on), and cells below this threshold stop oscillating (clock off). The clock is composed of the canonical Wnt and the Notch signaling pathways. Negative feedback mechanisms allow cells to switch once per clock cycle between Wnt signaling (blue; indicated as graded expression of Axin2 along the psm) and Notch signaling (salmon; indicated by Lfng expression). The segment boundary position is set at the interface between cells in the "clock on" and "clock off" state, which is determined by the threshold of the Wnt3a gradient during the Wnt signaling phase (the permissive state) of the clock cycle. Wnt controls Notch signaling in the clock, ensuring up-regulation of the latter in the same region down to the segment boundary position. A cascade of events leading to somite boundary formation is induced by Notch at this interface.

portant for the range in which Wnt signaling is active. Impairment of Notch signaling would affect the formation of sharp somite boundaries, whereas segmentation as such would proceed, possibly under control of other Wnt targets. Support for this model comes from the fact that Notch signaling is dispensable for segmentation, but not for the formation of regular and sharp somite boundaries. Wnt3a might employ the function of Notch for producing sharp somite boundaries exactly at the interface between neighboring segments. Additional experimental evidence is required to further substantiate the central components of this model.

\section{The primitive streak and tail bud-patterning centers} for axial development

The primitive streak and tail bud are not simply the source of cells for the psm. In these structures, mesenchymal cells with different developmental fate are generated, the gradient and the clock originate, and cells are endowed with information on their position along the A-P body axis. Wnt signaling appears to be involved in all of these events.

When Wnt3a expression is down-regulated in the tail bud, patterning of the psm is impaired. Segmentation is 

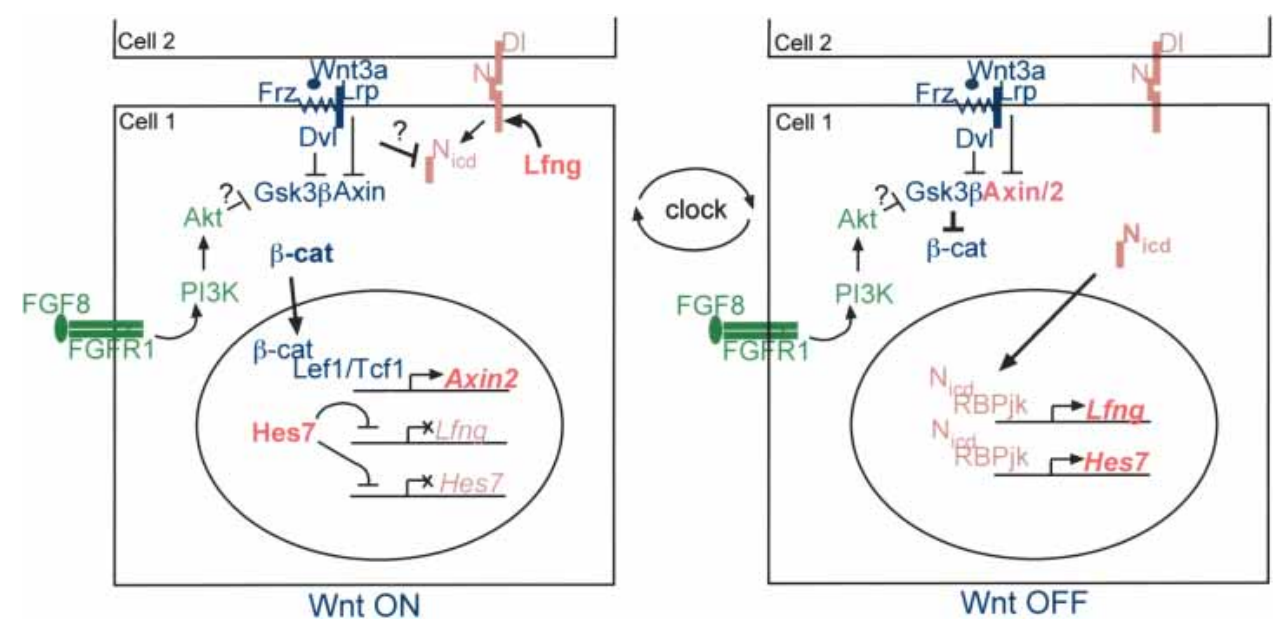

Figure 3. Model of the molecular clockwork. The clock is driven by Wnt signaling downstream of Wnt3a and is enhanced by FGF signaling through the PI-3 kinase pathway (green). Stabilization of $\beta$-catenin leads to up-regulation of Wnt targets (Wnt on). Axin2 is expressed, increasing the pool of Axin/Axin2, which results in negative feedback inhibition of the Wnt signaling pathway (blue) and concomitant activation of the Notch signaling pathway (salmon; Wnt off). The Notch targets Lfng and Hes7, among others, are expressed. Degradation of Axin2 RNA and destabilization of Axin2 protein reverse the process: Wnt signaling to the nucleus is re-established and Notch signaling is again inhibited (Wnt on), while Hes7 protein down-regulates Notch targets. The pool of activated Notch receptor (Nicd) is replenished through the action of Lfng and the Delta signal (Dl). Degradation of Hes7 protein ensures that the Notch targets can be expressed again. Highly unstable or transient products are labeled red, bold symbols indicate increased levels of the factor, and italicized symbols represent transcripts.

irregular and finally arrested (Greco et al. 1996; Aulehla et al. 2003). Eventually, psm formation in the tail bud and axial outgrowth cease (Greco et al. 1996).

Psm cells are endowed with positional information long before the segments actually form. Manifestation of this patterning event becomes morphologically clearly visible on formation of vertebrae of different shape (cervical, thoracal, lumbar, or sacral) from these segments (Kieny et al. 1972). Axial specification is directly controlled by the action of Hox genes (Kmita and Duboule 2003). Mutations in Hox genes lead to homeotic transformations: Segments (vertebrae) acquire a more anterior or more posterior identity. Hox gene activation is, at least in part, controlled by Notch signaling and linked to the segmentation clock, ensuring up-regulation of Hox genes in the appropriate segment (Dubrulle et al. 2001; Zakany et al. 2001). However, there is also good evidence for a link of the Wnt signal cascade to Hox gene expression. Mice carrying mutations of Wnt3a show homeotic transformations of the vertebrae, reminiscent of mutations in Hox genes (Ikeya and Takada 2001). In addition, $C d \times 1$, a direct target of the canonical Wnt signaling cascade, is involved in Hox gene activation and A-P patterning (Prinos et al. 2001; Lickert and Kemler 2002; for review, see Lohnes 2003). Thus, Wnt signaling appears to be involved in the control of Hox genes via Cdx transcription factors.

Interestingly, Wnt3a and its targets Brachyury, which is required for mesoderm formation, and $C d x 1$ all act in a dosage-dependent manner, increasing levels being required along the A-P axis (Stott et al. 1993; Greco et al. 1996; van den Akker et al. 2002). An increase of Wnt3a in the primitive streak and tail bud over time might control stepwise derepression of Hox genes, conferring positional information to the cells. By controlling Notch signaling in an indirect manner, Wnt signaling would also ensure up-regulation of Hox genes in the appropriate segments. Thus, Wnt signaling might control A-P patterning altogether.

It is quite clear that some important pieces of this puzzle are still missing, though the overall picture is taking shape. In our opinion, the available evidence is sufficient to postulate that Wnt signaling downstream of Wnt3a may be the prime factor controlling paraxial mesoderm formation, axial elongation, positional information, and segmentation in a highly integrated manner.

\section{Acknowledgments}

We thank Stephan Gasca, Ralf Spörle, and Lars Wittler for critical comments on the manuscript.

\section{References}

Aulehla, A. and Johnson, R.L. 1999. Dynamic expression of lunatic fringe suggests a link between notch signaling and an autonomous cellular oscillator driving somite segmentation. Dev. Biol. 207: 49-61.

Aulehla, A., Wehrle, C., Brand-Saberi, B., Kemler, R., Gossler, A., Kanzler, B., and Herrmann, B.G. 2003. Wnt3a plays a major role in the segmentation clock controlling somitogenesis. Dev. Cell 4: 395-406.

Axelrod, J.D., Matsuno, K., Artavanis-Tsakonas, S., and Perrimon, N. 1996. Interaction between Wingless and Notch signaling pathways mediated by dishevelled. Science 271: 1826-1832. 
Barrantes, I.B., Elia, A.J., Wunsch, K., De Angelis, M.H., Mak, T.W., Rossant, J., Conlon, R.A., Gossler, A., and de la Pompa, J.L. 1999. Interaction between notch signalling and lunatic fringe during somite boundary formation in the mouse. Curr. Biol. 9: 470-480.

Behrens, J., Jerchow, B.A., Wurtele, M., Grimm, J., Asbrand, C., Wirtz, R., Kuhl, M., Wedlich, D., and Birchmeier, W. 1998. Functional interaction of an axin homolog, conductin, with $\beta$-catenin, APC, and GSK3 $\beta$. Science 280: 596-599.

Bessho, Y. and Kageyama, R. 2003. Oscillations, clocks and segmentation. Curr. Opin. Genet. Dev. 13: 379-384.

Bessho, Y., Miyoshi, G., Sakata, R., and Kageyama, R. 2001. Hes7: A bHLH-type repressor gene regulated by Notch and expressed in the presomitic mesoderm. Genes Cells 6: 175185.

Bessho, Y., Hirata, H., Masamizu, Y., and Kageyama, R. 2003. Periodic repression by the bHLH factor Hes7 is an essential mechanism for the somite segmentation clock. Genes \& Dev. 17: 1451-1456.

Christ, B. and Ordahl, C.P. 1995. Early stages of chick somite development. Anat. Embryol. (Berl) 191: 381-396.

Christ, B., Jacob, H.J., and Jacob, M. 1974. Somitogenesis in the chick embryo. Determination of the segmentation direction. Verh. Anat. Ges. 68: 573-579.

Cole, S.E., Levorse, J.M., Tilghman, S.M., and Vogt, T.F. 2002. Clock regulatory elements control cyclic expression of Lunatic fringe during somitogenesis. Dev. Cell 3: 75-84.

Conlon, R.A., Reaume, A.G., and Rossant, J. 1995. Notch1 is required for the coordinate segmentation of somites. Development 121: 1533-1545.

Cooke, J. 1975. Control of somite number during morphogenesis of a vertebrate, Xenopus laevis. Nature 254: 196-199.

Cooke, J. and Zeeman, E.C. 1976. A clock and wavefront model for control of the number of repeated structures during animal morphogenesis. J. Theor. Biol. 58: 455-476.

Dale, K.J. and Pourquie, O. 2000. A clock-work somite. Bioessays 22: $72-83$.

Dale, J.K., Maroto, M., Dequeant, M.L., Malapert, P., McGrew, M., and Pourquie, O. 2003. Periodic notch inhibition by lunatic fringe underlies the chick segmentation clock. Nature 421: 275-278.

Dubrulle, J. and Pourquie, O. 2004. fgf8 mRNA decay establishes a gradient that couples axial elongation to patterning in the vertebrate embryo. Nature 427: 419-422.

Dubrulle, J., McGrew, M.J., and Pourquie, O. 2001. FGF signaling controls somite boundary position and regulates segmentation clock control of spatiotemporal Hox gene activation. Cell 106: 219-232.

Evrard, Y.A., Lun, Y., Aulehla, A., Gan, L., and Johnson, R.L. 1998. lunatic fringe is an essential mediator of somite segmentation and patterning. Nature 394: 377-381.

Fanto, M. and Mlodzik, M. 1999. Asymmetric Notch activation specifies photoreceptors R3 and R4 and planar polarity in the Drosophila eye. Nature 397: 523-526.

Flint, O.P., Ede, D.A., Wilby, O.K., and Proctor, J. 1978. Control of somite number in normal and amputated mutant mouse embryos: An experimental and a theoretical analysis. J. Embryol. Exp. Morphol. 45: 189-202.

Foltz, D.R., Santiago, M.C., Berechid, B.E., and Nye, J.S. 2002. Glycogen synthase kinase- $3 \beta$ modulates notch signaling and stability. Curr. Biol. 12: 1006-1011.

Forsberg, H., Crozet, F., and Brown, N.A. 1998. Waves of mouse Lunatic fringe expression, in four-hour cycles at two-hour intervals, precede somite boundary formation. Curr. Biol. 8: 1027-1030.

Fukumoto, S., Hsieh, C.M., Maemura, K., Layne, M.D., Yet,
S.F., Lee, K.H., Matsui, T., Rosenzweig, A., Taylor, W.G., Rubin, J.S., et al. 2001. Akt participation in the Wnt signaling pathway through Dishevelled. J. Biol. Chem. 276: $17479-17483$.

Galceran, J., Farinas, I., Depew, M.J., Clevers, H., and Grosschedl, R. 1999. Wnt3 $\mathrm{a}^{-/-}$-like phenotype and limb deficiency in Lef1 ${ }^{-/-} \mathrm{Tcf1}^{-/-}$mice. Genes \& Dev. 13: 709-717.

Greco, T.L., Takada, S., Newhouse, M.M., McMahon, J.A., McMahon, A.P., and Camper, S.A. 1996. Analysis of the vestigial tail mutation demonstrates that Wnt-3a gene dosage regulates mouse axial development. Genes \& Dev. 10: 313 324.

Haines, N. and Irvine, K.D. 2003. Glycosylation regulates Notch signalling. Nat. Rev. Mol. Cell Biol. 4: 786-797.

Hirata, H., Yoshiura, S., Ohtsuka, T., Bessho, Y., Harada, T., Yoshikawa, K., and Kageyama, R. 2002. Oscillatory expression of the bHLH factor Hes1 regulated by a negative feedback loop. Science 298: 840-843.

Hrabe de Angelis, M., McIntyre, J.N., and Gossler, A. 1997. Maintenance of somite borders in mice requires the Delta homologue DII1. Nature 386: 717-721.

Ikeya, M. and Takada, S. 2001. Wnt-3a is required for somite specification along the anteroposterior axis of the mouse embryo and for regulation of cdx-1 expression. Mech. Dev. 103: 27-33.

Iulianella, A., Melton, K.R., and Trainor, P.A. 2003. Somitogenesis: Breaking new boundaries. Neuron 40: 11-14.

Jho, E.H., Zhang, T., Domon, C., Joo, C.K., Freund, J.N., and Costantini, F. 2002. Wnt/ $\beta$-catenin/Tcf signaling induces the transcription of Axin2, a negative regulator of the signaling pathway. Mol. Cell. Biol. 22: 1172-1183.

Jouve, C., Palmeirim, I., Henrique, D., Beckers, J., Gossler, A., Ish-Horowicz, D., and Pourquie, O. 2000. Notch signalling is required for cyclic expression of the hairy-like gene HES1 in the presomitic mesoderm. Development 127: 1421-1429.

Jun, T., Gjoerup, O., and Roberts, T.M. 1999. Tangled webs: Evidence of cross-talk between c-Raf-1 and Akt. Sci. STKE 1999: PE1.

Kerszberg, M. and Wolpert, L. 2000. A clock and trail model for somite formation, specialization and polarization. J. Theor. Biol. 205: 505-510.

Kieny, M., Mauger, A., and Sengel, P. 1972. Early regionalization of somitic mesoderm as studied by the development of axial skeleton of the chick embryo. Dev. Biol. 28: 142-161.

Kmita, M. and Duboule, D. 2003. Organizing axes in time and space; 25 years of colinear tinkering. Science 301: 331-333.

Lickert, H. and Kemler, R. 2002. Functional analysis of cisregulatory elements controlling initiation and maintenance of early Cdxl gene expression in the mouse. Dev. Dyn. 225: 216-220.

Liu, P., Wakamiya, M., Shea, M.J., Albrecht, U., Behringer, R.R., and Bradley, A. 1999. Requirement for Wnt3 in vertebrate axis formation. Nat. Genet. 22: 361-365.

Lohnes, D. 2003. The Cdx1 homeodomain protein: An integrator of posterior signaling in the mouse. Bioessays 25: $971-$ 980.

Lustig, B., Jerchow, B., Sachs, M., Weiler, S., Pietsch, T., Karsten, U., van de Wetering, M., Clevers, H., Schlag, P.M., Birchmeier, W., et al. 2002. Negative feedback loop of Wnt signaling through upregulation of conductin/axin2 in colorectal and liver tumors. Mol. Cell. Biol. 22: 1184-1193.

Martinez Arias, A. 1998. Interactions between Wingless and Notch during the assignment of cell fates in Drosophila. Int. J. Dev. Biol. 42: 325-333.

McGrew, M.J., Dale, J.K., Fraboulet, S., and Pourquie, O. 1998. The lunatic fringe gene is a target of the molecular clock 
linked to somite segmentation in avian embryos. Curr. Biol. 8: 979-982.

Meinhardt, H. 1986. Models of segmentation. In Somites in developing embryos (eds. R.E. Bellairs, A. Donald, and J.W. Lash), pp. 179-189. Plenum, New York.

Morales, A.V., Yasuda, Y., and Ish-Horowicz, D. 2002. Periodic Lunatic fringe expression is controlled during segmentation by a cyclic transcriptional enhancer responsive to notch signaling. Dev. Cell 3: 63-74.

Oka, C., Nakano, T., Wakeham, A., de la Pompa, J.L., Mori, C., Sakai, T., Okazaki, S., Kawaichi, M., Shiota, K., Mak, T.W., et al. 1995. Disruption of the mouse RBP-J $\kappa$ gene results in early embryonic death. Development 121: 3291-3301.

Packard Jr., D.S. 1978. Chick somite determination: The role of factors in young somites and the segmental plate. J. Exp. Zool. 203: 295-306.

Palmeirim, I., Henrique, D., Ish-Horowicz, D., and Pourquie, O. 1997. Avian hairy gene expression identifies a molecular clock linked to vertebrate segmentation and somitogenesis. Cell 91: 639-648.

Palmeirim, I., Dubrulle, J., Henrique, D., Ish-Horowicz, D., and Pourquie, O. 1998. Uncoupling segmentation and somitogenesis in the chick presomitic mesoderm. Dev. Genet. 23: $77-85$.

Prinos, P., Joseph, S., Oh, K., Meyer, B.I., Gruss, P., and Lohnes, D. 2001. Multiple pathways governing Cdx1 expression during murine development. Dev. Biol. 239: 257-269.

Ruel, L., Bourouis, M., Heitzler, P., Pantesco, V., and Simpson, P. 1993. Drosophila shaggy kinase and rat glycogen synthase kinase- 3 have conserved activities and act downstream of Notch. Nature 362: 557-560.

Saga, Y. and Takeda, H. 2001. The making of the somite: Molecular events in vertebrate segmentation. Nat. Rev. Genet. 2: 835-845.

Sato, Y., Yasuda, K., and Takahashi, Y. 2002. Morphological boundary forms by a novel inductive event mediated by Lunatic fringe and Notch during somitic segmentation. Development 129: 3633-3644.

Seidensticker, M.J. and Behrens, J. 2000. Biochemical interactions in the wnt pathway. Biochim. Biophys. Acta 1495: $168-182$.

Serth, K., Schuster-Gossler, K., Cordes, R., and Gossler, A. 2003. Transcriptional oscillation of lunatic fringe is essential for somitogenesis. Genes \& Dev. 17: 912-925.

Stott, D., Kispert, A., and Herrmann, B.G. 1993. Rescue of the tail defect of Brachyury mice. Genes \& Dev. 7: 197-203.

Takada, S., Stark, K.L., Shea, M.J., Vassileva, G., McMahon, J.A., and McMahon, A.P. 1994. Wnt-3a regulates somite and tailbud formation in the mouse embryo. Genes \& Dev. 8: $174-189$.

Tam, P.P. 1981. The control of somitogenesis in mouse embryos. J. Embryol. Exp. Morphol. (Suppl.) 65: 103-128.

Tolwinski, N.S. and Wieschaus, E. 2004. Rethinking WNT signaling. Trends Genet. 20: 177-181.

van den Akker, E., Forlani, S., Chawengsaksophak, K., de Graaff, W., Beck, F., Meyer, B.I., and Deschamps, J. 2002. Cdx1 and $\mathrm{Cdx} 2$ have overlapping functions in anteroposterior patterning and posterior axis elongation. Development 129: 21812193.

Wolpert, L. 1989. Positional information revisited. Development (Suppl.) 107: 3-12.

Yamamoto, H., Kishida, S., Uochi, T., Ikeda, S., Koyama, S., Asashima, M., and Kikuchi, A. 1998. Axil, a member of the Axin family, interacts with both glycogen synthase kinase $3 \beta$ and $\beta$-catenin and inhibits axis formation of Xenopus embryos. Mol. Cell. Biol. 18: 2867-2875.
Zakany, J., Kmita, M., Alarcon, P., de la Pompa, J.L., and Duboule, D. 2001. Localized and transient transcription of Hox genes suggests a link between patterning and the segmentation clock. Cell 106: 207-217.

Zeng, L., Fagotto, F., Zhang, T., Hsu, W., Vasicek, T.J., Perry III, W.L., Lee, J.J., Tilghman, S.M., Gumbiner, B.M., and Costantini, F. 1997. The mouse Fused locus encodes Axin, an inhibitor of the Wnt signaling pathway that regulates embryonic axis formation. Cell 90: 181-192.

Zhang, N. and Gridley, T. 1998. Defects in somite formation in lunatic fringe-deficient mice. Nature 394: 374-377. 


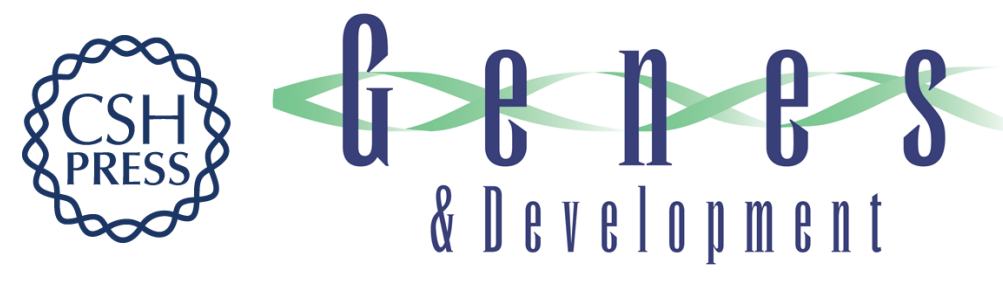

\section{Segmentation in vertebrates: clock and gradient finally joined}

Alexander Aulehla and Bernhard G. Herrmann

Genes Dev. 2004, 18:

Access the most recent version at doi:10.1101/gad.1217404

References This article cites 62 articles, 19 of which can be accessed free at: http://genesdev.cshlp.org/content/18/17/2060.full.html\#ref-list-1

License

Email Alerting Receive free email alerts when new articles cite this article - sign up in the box at the top Service right corner of the article or click here.

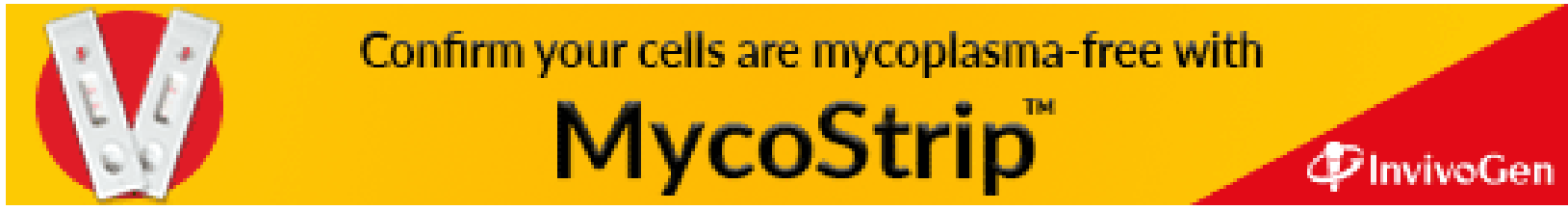

